\title{
BMC
}

Research Notes

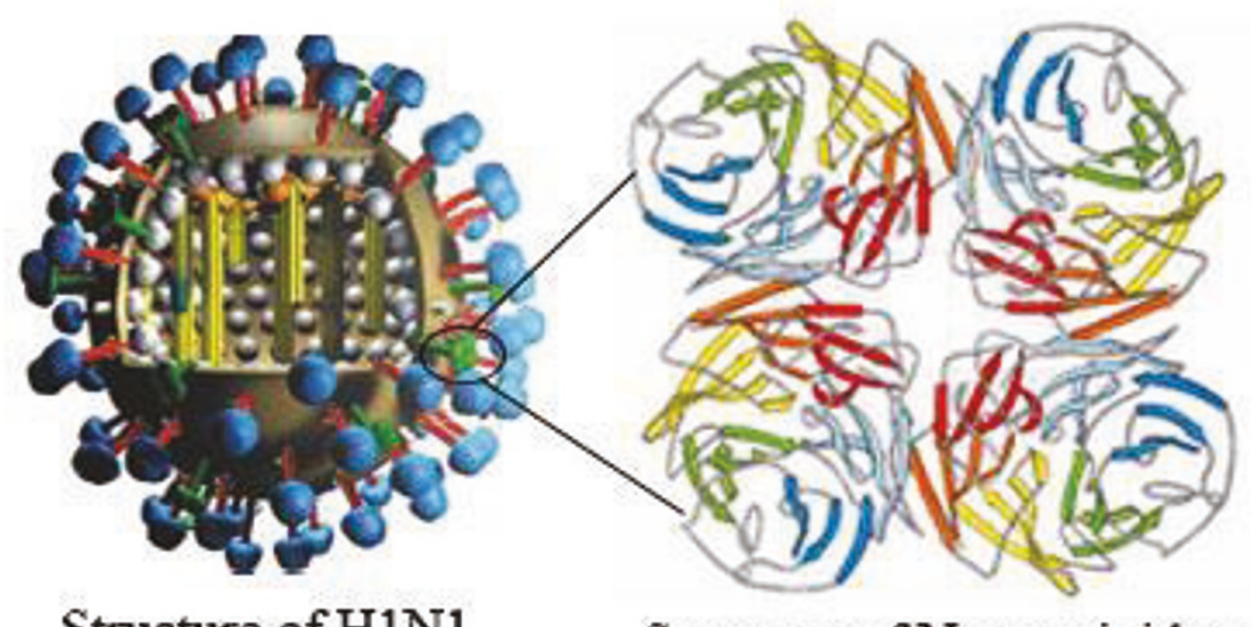

Structure of H1N1

Influenza strain

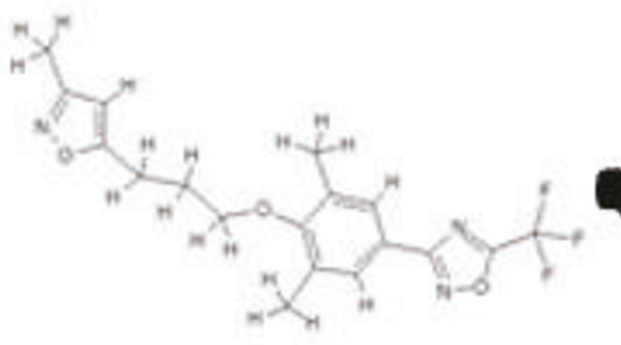

Structure of Pleconaril

$+$

Structure of Neuraminidase

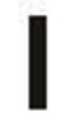

Active site analysis

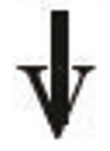

Docking

analysis

310 Novel substituted Pleconaril derivatives

\section{In-Silico screening of Pleconaril and its novel substituted derivatives with Neuraminidase of H1N1 Influenza strain}

Basha and Prasad

C Biomed Central

Basha and Prasad BMC Research Notes 2012, 5:105

http://www.biomedcentral.com/1756-0500/5/105 (17 February 2012) 


\title{
In-Silico screening of Pleconaril and its novel substituted derivatives with Neuraminidase of H1N1 Influenza strain
}

\author{
Syed Hussain Basha* and R Nalini Prasad
}

\begin{abstract}
Background: Neuraminidase (NA) is a prominent surface antigen of Influenza viruses, which helps in release of viruses from the host cells after replication. Anti influenza drugs such as Oseltamivir target a highly conserved active site of NA, which comprises of 8 functional residues (R118, D151, R152, R224, E276, R292, R371 and Y406) to restrict viral release from host cells, thus inhibiting its ability to cleave sialic acid residues on the cell membrane. Reports on the emergence of Oseltamivir resistant strains of H1N1 Influenza virus necessitated a search for alternative drug candidates. Pleconaril is a novel antiviral drug being developed by Schering-Plough to treat Picornaviridae infections, and is in its late clinical trials stage. Since, Pleconaril was designed to bind the highly conserved hydrophobic binding site on VP1 protein of Picorna viruses, the ability of Pleconaril and its novel substituted derivatives to bind highly conserved hydrophobic active site of H1N1 Neuraminidase, targeting which oseltamivir has been designed was investigated.
\end{abstract}

Result: 310 novel substituted variants of Pleconaril were designed using Chemsketch software and docked into the highly conserved active site of NA using arguslab software. 198 out of 310 Pleconaril variants analyzed for docking with NA active site were proven effective, based on their free binding energy.

Conclusion: Pleconaril variants with $\mathrm{F}, \mathrm{Cl}, \mathrm{Br}, \mathrm{CH} 3, \mathrm{OH}$ and aromatic ring substitutions were shown to be effective alternatives to Oseltamivir as anti influenza drugs.

Keywords: Pleconaril, Oseltamivir, H1N1, Neuraminidase, Docking analysis

\section{Background}

Most of the early antiviral drugs were discovered after screening large number of possible drug compounds using trial and error method. Lately, this approach has been largely replaced by rational drug design, in which, a target viral protein is identified for the drug [1]. A detailed picture of the 3 dimensional structure of the protein can be derived using In-silico computational techniques and a target site in the protein can be selected [2]. In influenza viruses, NA surface antigen plays a vital role in releasing the virus from the host cell during the budding stage [3]. Ever since the crystal structure of NA was determined, it is used as a target protein for many drug compounds. Oseltamivir (Figure 1) is one such

\footnotetext{
* Correspondence: hassainbasha53@gmail.com

Dept. of Biotechnology, REVA Institute of Science and Management, Yelahanka, Bangalore 560 064, India
} highly conserved NA active site of H1N1 Influenza virus which comprises of 8 functional residues (R118, D151, R152, R224, E276, R292, R371 and Y406) [4,5]. The recent outbreaks of $\mathrm{H} 1 \mathrm{~N} 1$ and reports of oseltamivir resistant strains have necessitated the need to find effective alternatives to the existing anti-influenza drugs [6].

Pleconaril (Figure 2) is a novel antiviral drug being developed by Schering-Plough to treat Picornaviridae infections, and is in its late clinical trials stage [7]. Highly conserved hydrophobic pocket of VP1 protein, which forms a part of picornaviral capsid, is the target for Pleconaril activity. In enteroviruses, this prevents the virus from exposing its RNA, and in rhinoviruses it prevents the virus from attaching itself to the host cell [8]. However, to date, to the best of our knowledge there is no study reporting the activity of Pleconaril against 


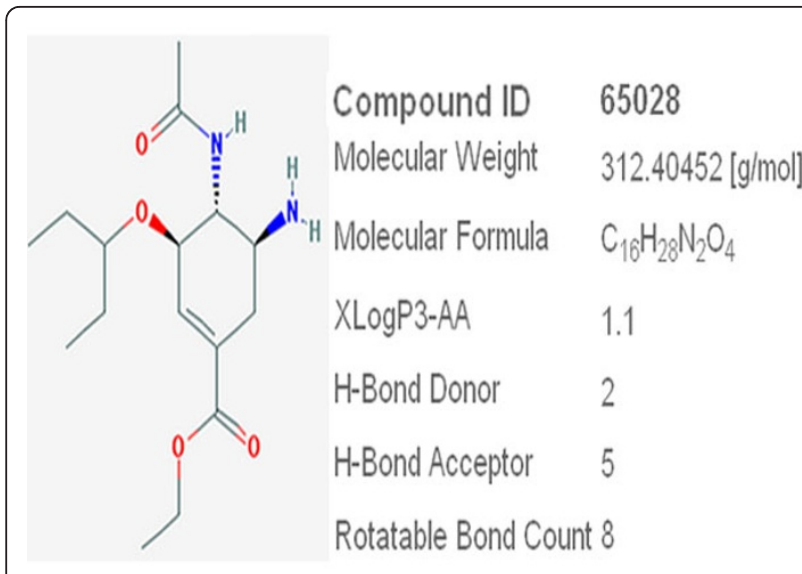

Figure 1 Structure and properties of Oseltamivir.

Influenza virus. Since, Pleconaril was designed to bind the highly conserved hydrophobic binding site on VP1 protein of Picorna viruses, the ability of Pleconaril and its novel substituted derivatives to bind highly conserved hydrophobic active site of H1N1 Neuraminidase, targeting which oseltamivir has been designed was investigated in this present study.

Molecular modeling tools help in generating new candidate drug molecules within a short span of time. After generation of new possible drug candidates, the drug and target protein interaction dynamics can be predicted by carrying out a docking analysis. The knowledge so derived is used to predict the strength of association or binding affinity between the two molecules based on scoring functions.

A database of potential drug molecules and target protein structure serve as inputs for docking analysis. The success of the docking analysis is determined by scoring function and search algorithm [9], which helps in determining the compatibility between the drug and its target

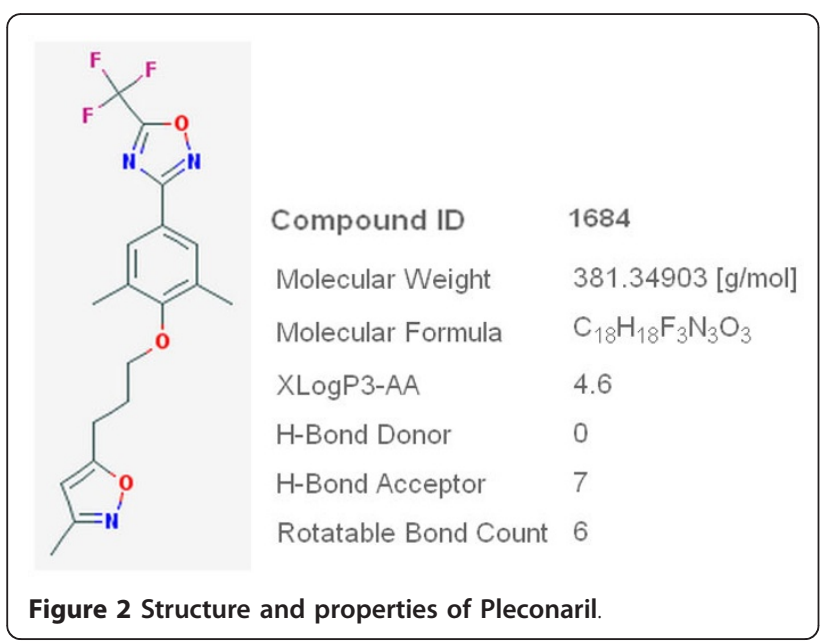

protein. This technique is being used extensively to predict the geometries of different bimolecular complexes [10]. Scoring function predicts the strength of the binding affinity between ligand and the protein based on the complex geometry [11] and search algorithm analyzes the drug molecule for different binding positions with its target molecule, each binding position, which is termed a "pose", is used to generate the snapshot of interactions [12].

In our present study, a database of 310 novel substituted Pleconaril variants was built by altering the side chains and substituting different aromatic rings into the original Pleconaril molecule. Molecular docking analysis was performed to visualize the interaction of each of these variants with target molecule. An attempt was made to identify Pleconaril variants with best NA binding ability.

\section{Methods}

\section{Preparation of Receptor}

The crystal structure of NA of 1918 Spanish flu (A/Brevig Mission/1/18 H1N1) virus (PDB ID: 3BEQ) was obtained from Protein Data Bank (PDB) [13] with a resolution factor of $1.64 \AA$ and the method incorporated is X-Ray diffraction [14]. Before docking, the crystal structure of the protein was cleaned by removing the water molecules and hydrogen atoms were added to this target protein for correct tautomeric and ionization states of amino acid residues. The modified structure so obtained was saved in .pdb format and used for all docking studies.

\section{Preparation of Ligands}

Oseltamivir (Compound ID 65028) [15] and Pleconaril (Compound ID 1684) [16] were obtained from pubchem database [17]. Using ACDLABS ChemSketch 11.0 [18] software, 310 novel substituted derivatives of Pleconaril were designed using $\mathrm{Cl}, \mathrm{F}, \mathrm{Br}, \mathrm{CH} 3$ and $\mathrm{OH}$ functional groups as substitutes on R, R1, R2, R3, R4, R5, and R6 positions of 11 basic Pleconaril variants (Figure 3).

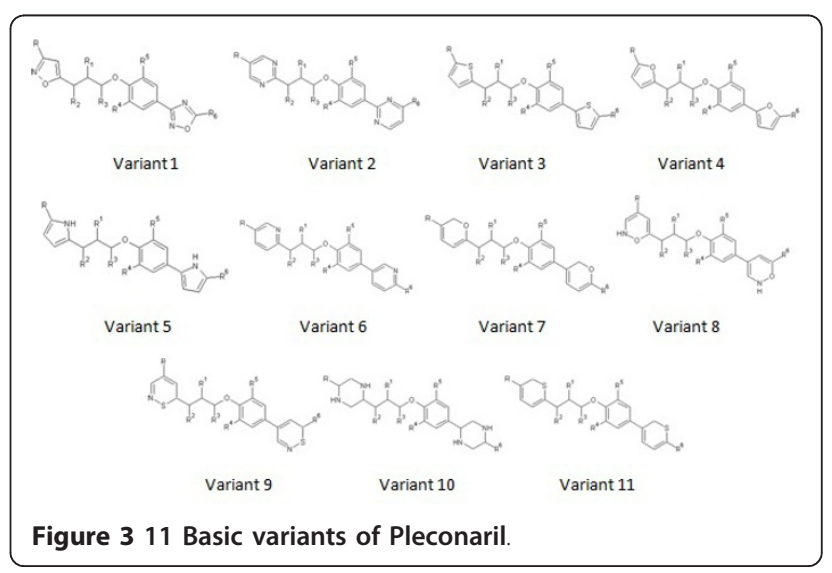




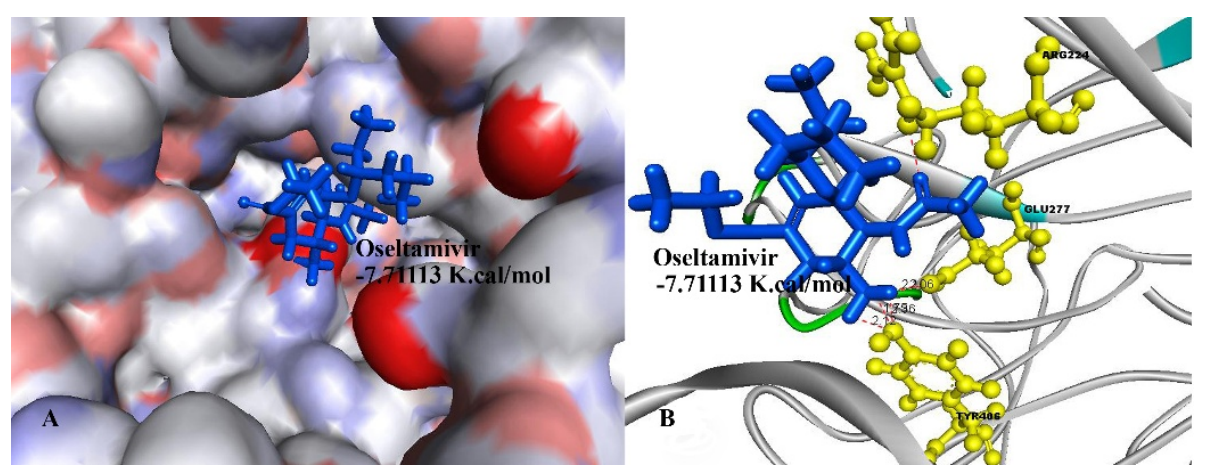

Figure 4 A) Binding of oseltamivir (Blue) into the active site of NA. B) Amino acid residues (Yellow) in the active site of NA interacting with Oseltamivir by hydrogen bonds (dotted red line) using $-7.71113 \mathrm{~K}$.Cal./mol. of binding energy.

Using Arguslab 4.0.1 [19] software, hydrogen bonds were added to each molecule and fidelity of all bonds was checked using "add hydrogens" and "Clean Hybridization" options respectively. Geometry optimization was done using UFF [20-24] Molecular Mechanics (MM) method. Finally Oseltamivir, Pleconaril and all the novel substituted Pleconaril derivatives were saved in .mol format for further docking studies.

\section{Determination of Active site}

The highly conserved active site of NA, which comprises of 8 functional residues (R118, D151, R152, R224, E276, R292, R371 and Y406) targeting which oseltamivir has been designed was considered as active site for docking analysis.

\section{Docking}

Docking between receptor and ligands was performed using "Dock a Ligand" option of arguslab 4.0.1 software. A spacing of $0.4 \AA$ between the grid points was used. "ArgusDock" was selected as docking engine. "Regular precision" was selected in docking precision menu, "Dock" was chosen as calculation type, "Flexible" for the ligand and "AScore" was used as the scoring function. A maximum of 150 poses were allowed to be analyzed, binding site box was set to $25 \times 25 \times 25$ angstroms to encompass the entire active site. Each docking run was repeated three times to get best results. Resulted docked molecules were saved in .pdb format and all the docking images were generated using Accelrys ${ }^{\circledR}$ Discovery Studio 3.0 Visualizer software [25].

\section{Results and discussion}

All the 310 variants of Pleconaril molecule were analyzed for binding with the active site of NA. 198 out of these were found to have optimum binding efficiency, based on the binding energy calculations in comparison with Oseltamivir.

Further investigations showed that Oseltamivir formed 6 hydrogen bonds with TYR 406, GLU 277, ARG 224 (Figure 4) and Pleconaril formed 6 hydrogen bonds with SER 246, PRO 245, ARG 118 amino acid residues (Figure 5), whereas the best Pleconaril variant formed 9 hydrogen bonds with ARG 118, ASN 347, ARG 371 and GLU 277 amino acid residues of NA active site. Moreover the central benzene ring and furan ring of the best Pleconaril variant played a major role in stabilizing the

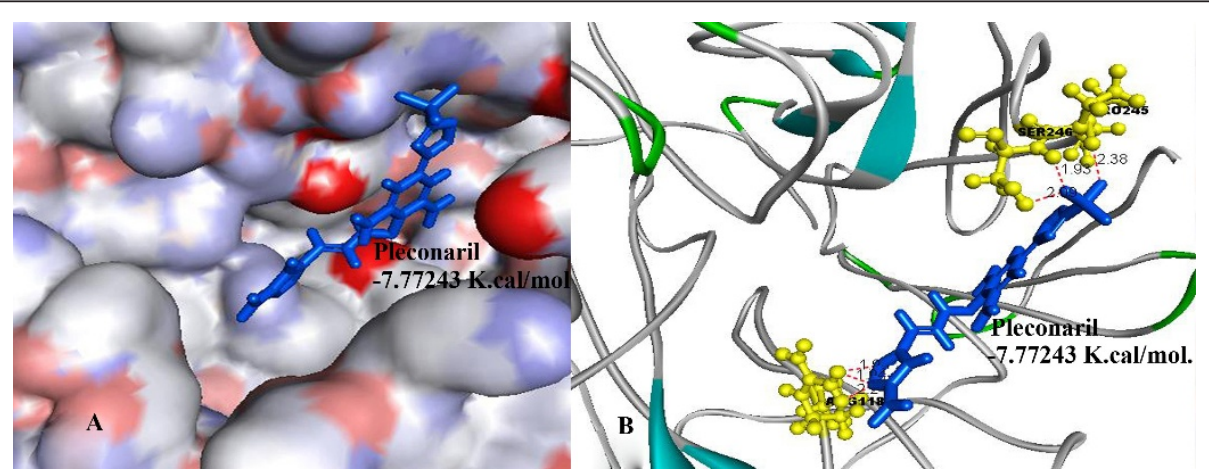

Figure 5 A) Binding of Pleconaril (Blue) into the active site of NA. B) Amino acid residues (Yellow) in the active site of NA interacting with Pleconaril by hydrogen bonds (dotted red line) using -7.77243 K.Cal./mol. of binding energy. 


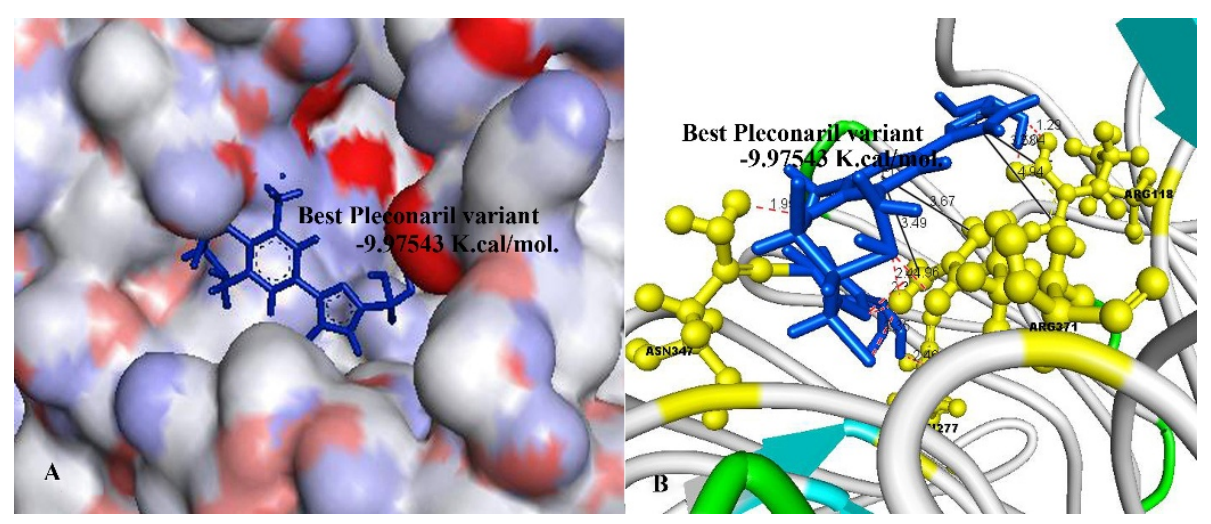

Figure 6 A) Binding of Best Pleconaril variant (Blue) into the active site of NA. B) Amino acid residues (Yellow) in the active site of NA interacting with Best Pleconaril variant by hydrogen bonds (dotted red line) and pi-cationic interactions (Solid Black line) using -9.97543 K.Cal./ mol. of binding energy.

ligand receptor complex by pi-cation interactions with amino acid residues ARG 118 and ARG 371 along with hydrogen bonds (Figure 6).

Pleconaril derivative (Numbered 3 in Table 1), with a binding energy of $-9.97543 \mathrm{~K} . \mathrm{cal} / \mathrm{mol}$. showed the least possible binding energy of all variants analyzed. Whereas the binding energy of oseltamivir, the original NA inhibitor, was found to be $-7.7113 \mathrm{~K} . \mathrm{cal} / \mathrm{mol}$. 44 variants of Pleconaril were found to be having binding energy around $-9.0 \mathrm{~K} . \mathrm{cal} / \mathrm{mol}$. 10 best variants out of

Table 1 Structure, molecular formula, binding energies of Oseltamivir, Pleconaril and best variant of pleconaril

S. No<smiles>Cc1cc(-c2ccc(C(O)(O)O)o2)ccc1OC(O)C(O)C(O)c1ccc(C(O)(O)O)o1</smiles> 
Basho and Prasad BMC Research Notes 2012, 5:105

Page 5 of 7

http://www.biomedcentral.com/1756-0500/5/105

Table 2 Structure, molecular formula, binding energies of 10 best variants of pleconaril

S. No

Structure of molecule

Molecular formula

Binding energy in K.cal./mol.

1.<smiles>Cc1ccc(C(Cl)C(Cl)C(Cl)Oc2c(C)cc(-c3ccc(C(O)(O)O)s3)cc2C)s1</smiles>

$\mathrm{C}_{21} \mathrm{H}_{21} \mathrm{Cl}_{3} \mathrm{O}_{4} \mathrm{~S}_{2}$

$-9.90775$

2.

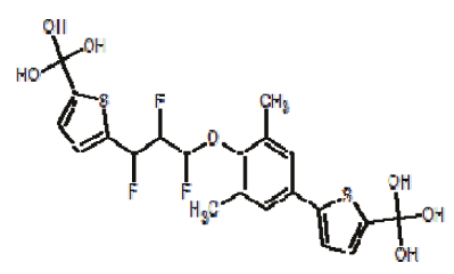

$\mathrm{C}_{21} \mathrm{H}_{21} \mathrm{~F}_{3} \mathrm{O}_{7} \mathrm{~S}_{2}$

$-9.90392$

3.

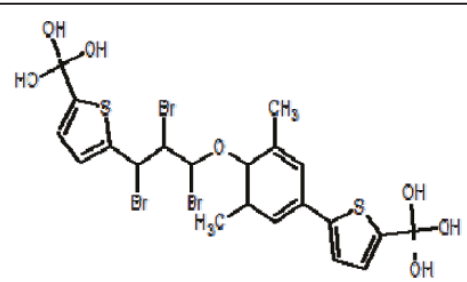

$\mathrm{C}_{21} \mathrm{H}_{21} \mathrm{Br}_{3} \mathrm{O}_{7} \mathrm{~S}_{2}$

$-9.90239$

4.

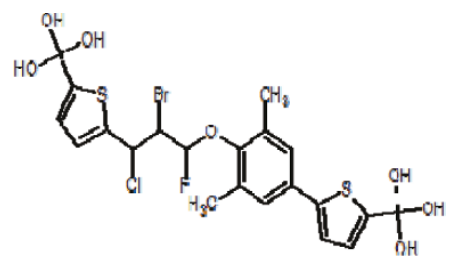

$\mathrm{C}_{21} \mathrm{H}_{21} \mathrm{BrClFO}_{7} \mathrm{~S}_{2}$

$-9.69162$

5.

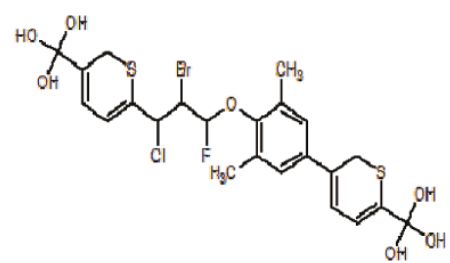

$\mathrm{C}_{23} \mathrm{H}_{25} \mathrm{BrClFO}_{7} \mathrm{~S}_{2}$

$-9.67661$

6.

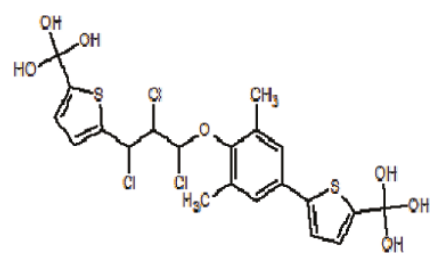

$\mathrm{C}_{21} \mathrm{H}_{21} \mathrm{Cl}_{3} \mathrm{O}_{7} \mathrm{~S}_{2}$

$-9.60862$

7.

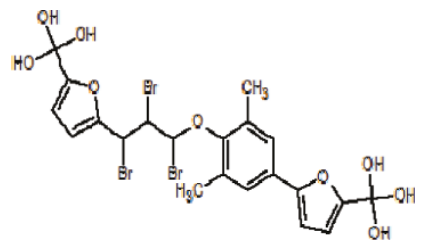

$\mathrm{C}_{21} \mathrm{H}_{21} \mathrm{Br}_{3} \mathrm{O}_{9}$

$-9.57821$ 
Table 2 Structure, molecular formula, binding energies of 10 best variants of pleconaril (Continued)

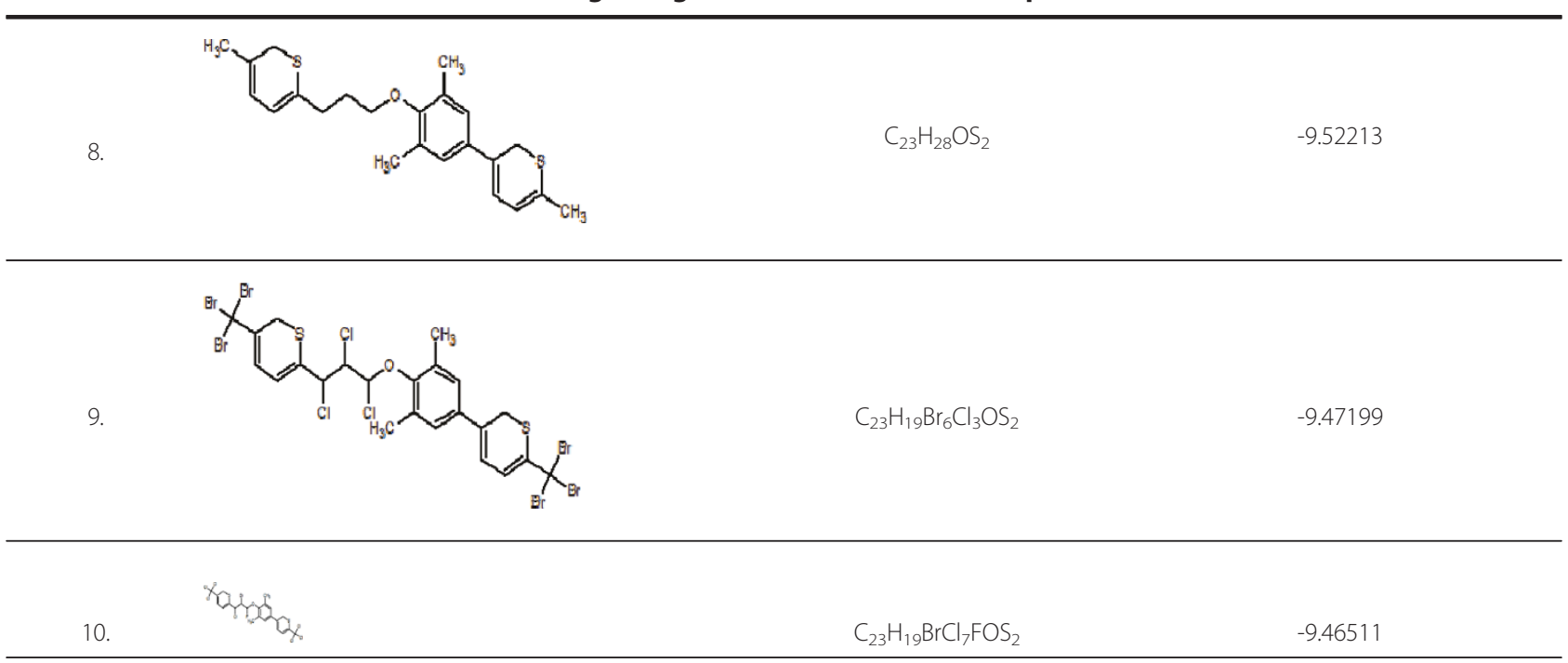

these 44 were tabulated in Table 2 . These molecules were selected because of their least binding energies, as the drug molecules with the least binding energy are generally considered to be having high binding efficiencies. Analysis of these 44 variants in further established that furan and thiophene substitutions along with the presence of central benzene ring in the main molecule played an important role in determining the binding affinity of the drug variants.

\section{Conclusion}

Computer aided drug designing and molecular docking analysis are highly effective in creating and analyzing new candidate drug molecules. 198 out of 310 Pleconaril variants analyzed for docking with NA active site were proven effective. Pleconaril variants with $\mathrm{F}, \mathrm{Br}, \mathrm{CH} 3, \mathrm{Cl}, \mathrm{OH}$ and aromatic ring substitutes showed higher levels of NA binding ability. Several interactions such as hydrogen bonds, hydrophobic, hydrophilic interactions, electrostatics and Vanderwaal forces are thought to have played an important role in stabilizing the drug and target complexes based on the theoretical modeling. Thus, based on the above results we propose Pleconaril variants numbered 3, 4 and 11 (Figure 4) with $\mathrm{F}, \mathrm{Br}, \mathrm{CH}, \mathrm{Cl}$ and $\mathrm{OH}$ substitutions at R, R1, R2, R3, R4, R5, and R6 positions have a definite potential to be developed as lead compounds for $\mathrm{H} 1 \mathrm{~N} 1$ Influenza virus. However as it is only a preliminary In-silico investigation in modifying the Pleconaril molecule for anti-influenza activity, further In-vivo validation and conformation of the present findings is required.

\section{Acknowledgements}

The authors wish to thank Dr. N. Ramesh Principal, REVA ISM for extending his help for using the computational facilities at the Dept. of Biotechnology, REVA ISM. T.P. Charanraj, Lecturer, Dept. of Biochemistry, REVA ISM for extending his help in designing the novel substituted Pleconaril derivatives and Prof. H. Junjappa, Professor of Synthetic organic chemistry, REVA ISM for his valuable insights in analyzing and interpretation of the data.

\section{Authors' contributions}

SHB conceptualized the idea, carried out the work including acquisition of the data, analysis of the final results and drafting the manuscript. RNP was involved in supervising the work designed, analysis and interpretation of the data as well as drafting the manuscript. Both authors read and approved the final manuscript.

\section{Competing interests}

The authors declare that they have no competing interests.

Received: 6 September 2011 Accepted: 17 February 2012

Published: 17 February 2012

\section{References}

1. Noble D, Colatsky TJ: A return to rational drug discovery: computerbased models of cells, organs and systems in drug target identification. Emerging therapeutic targets 2000, 4:39-49.

2. Ekins S, Mestres J, Testa B: In-silico pharmacology for drug discovery: Applications to targets and beyond. Br J Pharmacol 2007, 152:21-37.

3. John Cartar B, Venetia Saunders A: Virology-principles and applications. India: John Wiley and sons limited publications; First 2007, 317-322.

4. Von Itzstein M, Wu WY, Kok GB, Pegg MS, Dyason JC, et al: Rational design of potent sialidase-based inhibitors of influenza virus replication. Nature 1993, 363:418-423.

5. Aoki FY, Boivin G, Roberts NA: Influenza virus susceptibility and resistance to Oseltamivir. Anti viral Therapy 2007, 12:603-616.

6. Magden J, Kääriäinen L, Ahola T: Inhibitors of virus replication:recent developments and prospects. Appl Microbiol Biotechnol 2005, 66:612-621.

7. Effects of Pleconaril Nasal Spray on Common Cold Symptoms and Asthma Exacerbations Following Rhinovirus Exposure (Study P04295AM2). ClinicalTrials.gov. U.S. National Institutes of Health; 2007, Retrieved 2011-12-26.

8. Florea N, Maglio D, Nicolau D: Pleconaril, a novel antipicornaviral agent. Pharmacotherapy 2003, 23(3):339-48.

9. Kitchen DB, Decornez H, Furr JR, Bajorath J: Docking and scoring in virtual screening for drug discovery: Methods and applications. Nature Review Drug Discovery 2004, 3:935-949.

10. Irawin Kuntz D, Elaine Meng C, Shoichet Brain K: Structure-Based Molecular Design. Accounts of Chemical research 1994, 27(5):117-123.

11. Jain AN: Scoring functions for protein-ligand docking. Curr Protein Pept Sci 2006, 7(5):407-20. 
12. Shoichet BK, Kuntz ID, Bodian DL: Molecular docking using shape descriptors. J Comput Chem 2004, 13(3):380-397.

13. Bernstein FC, Koetzle TF, Williams GJ, Meyer EE Jr, Brice MD, Rodgers JR, Kennard O, Shimanouchi T, Tasumi M: The Protein Data Bank: A Computer-based Archival File For Macromolecular Structures. J Mol Biol 1977, 112:535.

14. Xiaojin Xu, Xueyong Zhu, Raymond Dwek A, James Stevens, lan Wilson A: Structural Characterization of the 1918 Influenza Virus H1N1 Neuraminidase. J Virol 2008, 82(21):10493-10501.

15. , National Center for Biotechnology Information: PubChem Compound Database; CID = 65028, http://pubchem.ncbi.nlm.nih.gov/summary/ summary.cgi? cid=65028\&loc=ec_rcs (accessed Dec. 26, 2011).

16. , National Center for Biotechnology Information: PubChem Compound Database; CID = 1684, http://pubchem.ncbi.n/m.nih.gov/summary/summary. cgi?cid=1684\&loc=ec_rcs (accessed Dec. 26, 2011).

17. Bolton E, Wang Y, Thiessen PA, Bryant SH: PubChem: Integrated Platform of Small Molecules and Biological Activities. In Chapter 12 IN Annual Reports in Computational Chemistry. Volume 4. American Chemical Society, Washington, DC; 2008

18. 2007, ACD/ChemSketch Freeware, version 11.01, Advanced Chemistry Development, Inc., Toronto, ON, Canada, www.acdlabs.com.

19. Mark A: Thompson, ArgusLab 4.0.1, Planaria Software LLC, Seattle, WA [http://www.arguslab.com]

20. Rappe AK, Casewit CJ, Colwell KS, Goddard WA, Skiff WM: UFF, a full periodic table force field for molecular mechanics and molecular dynamics simulations. J Am Chem Soc 1992, 114:10024-10035.

21. Rappe AK, Colwell KS, Casewit CJ: Application of a Universal force field to metal complexes. Inorg Chem 1993, 32:3438-3450.

22. Rappe AK, Goddard WA: Charge Equilibration for molecular dyanamics simulations. J Phys Chem 1991, 95:3358-3363.

23. Casewit CJ, Colwell KS, Rappe' AK: Application of a universal force field to organic molecules. J Am Chem Soc 1992, 114:10035-10046.

24. Casewit CJ, Colwell KS, Rappe' AK: Application of a universal force field to main group compounds. J Am Chem Soc 1992, 114:10046-10053.

25. , Accelrys Software Inc., 2011, Discovery studio Visualizer 3.0, http://accelrys com/products/discovery-studio/visualization-download.php.

doi:10.1186/1756-0500-5-105

Cite this article as: Basha and Prasad: In-Silico screening of Pleconaril and its novel substituted derivatives with Neuraminidase of $\mathrm{H} 1 \mathrm{~N} 1$ Influenza strain. BMC Research Notes 2012 5:105.

\section{Submit your next manuscript to BioMed Central and take full advantage of:}

- Convenient online submission

- Thorough peer review

- No space constraints or color figure charges

- Immediate publication on acceptance

- Inclusion in PubMed, CAS, Scopus and Google Scholar

- Research which is freely available for redistribution

Submit your manuscript at www.biomedcentral.com/submit 